\title{
Motivos de rechazo emitidos por el alumnado del primer ciclo de educación primaria de Castellón, Palma de Mallorca, Sevilla y Valladolid
}

\author{
Aida Sanahuja Ribés \\ al106899@uji.es \\ Francisco Juan García Bacete \\ fgarcia@uji.es \\ Ghislaine Marande Perrin \\ marande@uji.es \\ Andrea Rubio Barreda \\ andrea.rubio@uji.es \\ Inés Milián Rojas \\ al061904@uji.es \\ Sara Roselló Sempere \\ al258700@uji.es
}


Agradecimientos: La realización de este artículo ha sido posible gracias a las ayudas del Ministerio de Economía y Competitividad (Referencia EDU2012-35930), por la Fundación Bancaja-Universitat Jaume I (Referencia P11A2012-04) y por la Fundación Dávalos-Fletcher de Castellón (convocatoria de 2009). 
Los investigadores se preguntan por qué los niños ${ }^{1}$ se aceptan y se rechazan y cuáles son las razones que emiten para aceptar o rechazar a un compañero. Según la literatura diversos estudios se han centrado en la categorización de los motivos de aceptación y rechazo, pero ninguno en las diferencias existentes en la emisión de motivos de rechazo según la zona geográfica de procedencia.

El objetivo principal de este artículo es ver si los alumnos de Castellón de la Plana, Palma de Mallorca, Sevilla y Valladolid presentan un perfil diferente en la emisión de motivos de rechazo. Para identificar los motivos de rechazo se ha administrado el cuestionario sociométrico de nominación entre iguales. La muestra está formada por un total de 939 alumnos del primer ciclo de educación primaria, escolarizados en 40 aulas de centros públicos de España. A partir de dicho instrumento se han recogido un total de 2.965 respuestas como motivos de rechazo y a partir de éstas se ha constituido una taxonomía formada por 6 categorías y 7 subcategorías.

Los resultados muestran que sí que existen diferencias significativas en función de la zona de procedencia del emisor. Los alumnos de Castellón, Palma de Mallorca, Sevilla y Valladolid presentan un perfil diferente en la emisión de razones de rechazo, y por ello, existen diferencias culturales entre las diferentes zonas.

Palabras clave: Rechazo entre iguales, motivos de rechazo, categorización, sociometria, primer ciclo de educación primaria.

\section{Introducción}

Nuestro foco de atención se centra en las relaciones entre iguales que se producen en el aula. La expresión "relaciones entre iguales» (del inglés peers) se utiliza para referirse a las interacciones niño-niño porque su principal característica es que quienes interactúan tienen edades, intereses, características, roles, etc. iguales o muy parecidos (Monjas, 2007). También se denominan relaciones entre pares, interacciones entre compañeros, relaciones entre colegas o relaciones con coetáneos (García Bacete y González, 2010).

Todo el mundo está de acuerdo en la importancia de las relaciones entre iguales en el desarrollo de las personas. El clásico argumento formulado por Hartup (1983) es que los niños a partir de los cuatro años dedican cada vez más tiempo a estar con sus pares. Sin duda,

${ }^{1}$ Cada vez que se mencione a niños, compañeros, alumnos, ... se entiende que se hace referencia a ambos sexos indistintamente. 
actualmente este argumento se ve reforzado porque los niños se incorporan a las escuelas infantiles mucho antes de los cuatro años y se reconoce cada vez más la importancia de las relaciones entre iguales en la juventud, durante la vida adulta e incluso durante la vejez. Los iguales dan forma a un importante contexto social en el que se aprenden muchas habilidades y procesos que son esenciales para la adaptación y ajuste de las personas (García Bacete, Sureda y Monjas, 2010).

La aceptación social, es entendida como el grado en que un niño es querido, aceptado, reconocido y apreciado en su grupo de iguales, está asociada con la buena adaptación mientras que la baja aceptación social, la ignorancia y el rechazo son factores de riesgo importantes para la desadaptación presente y futura (Bukowski, Newcomb y Hartup, 1996). El que un niño sea aceptado significa que los compañeros buscan su compañía y su amistad y que le estiman, le valoran y le quieren, mientras que la ignorancia, y especialmente el rechazo, denotan desde falta de estima, de atracción y de valoración hasta antipatía y desagrado (Suerda, García Bacete y Monjas, 2009).

Una pregunta que los estudiosos se hacen repetidamente es por qué los niños se aceptan y se rechazan, es decir, cuáles son las razones que ellos mismos dan para elegir a un compañero y preferir relacionarse con él y cuáles arguyen para explicitar su desagrado y disgusto para relacionarse con otros (Williams y Asher, 1993). Varios investigadores han centrado su estudio en los motivos y razones esgrimidas por los niños para rechazar a sus compañeros (Rogosch y Newcombs, 1989; Williams y Asher, 1993; Monjas, Sureda y García Bacete, 2008; García Bacete y Sanahuja, 2013).

Rogosch y Newcombs (1989) realizaron un estudio en el que participaron 182 alumnos de 7, 8 y 10 años. Con él concluyeron que los niños se rechazan principalmente por: atractivo físico negativo; pobres habilidades físicas; funcionamiento pobre del aula; exclusión activa; ser inmaduro; ser extraño; ser blanco de las bromas de los demás; ser repugnante, mezquino, desleal; ser engreído, mandón; ser el payaso de la clase y a menudo metrese en problemas; ser agresivo físicamente; ser consentido, mocoso; no gustar; excluir a los otros; novios / novias y ser niños o niñas perseguidores.

Williams y Asher (1993), en su trabajo Las razones para el rechazo entre iguales, se preguntan qué buscan los niños en su relación con los iguales $\mathrm{y}$ por qué unos niños son aceptados $\mathrm{y}$ otros rechazados $\mathrm{y}$ comentan que los niños, a la hora de decidir si hacerse amigos de alguien, se hacen a sí mismos unas preguntas esenciales:

«¿Es divertido estar con este niño?, ¿Nos influimos mutuamente en la dirección que a mí me gusta?, Este niño, ¿es digno de confianza?, ¿Me ayuda a conseguir mis metas?, ¿Me hace sentir bien? y ¿Es similar a mí?» 
Por supuesto, los niños rara vez piensan en estas cuestiones de manera consciente. Sin embargo, los investigadores indican que las respuestas a estas preguntas determinan si los niños van a aceptar o no a un niño. Pero, ¿̇los niños cómo deciden que alguien es divertido o es digno de confianza? Una base importante de esta decisión es el comportamiento que se observa en el otro niño.

Monjas, Sureda y García Bacete (2008), con su estudio determinaron que los niños de 10 y 11 años rechazan a sus compañeros principalmente por: la "dominancia y superioridad» (categoría 12 ) con un $21,4 \%$ de las respuestas; en particular, la "prepotencia» es el motivo que obtiene porcentajes de respuesta más altos (14,8\%). A continuación, encontramos un bloque de motivos que aporta cerca del $10 \%$ de las respuestas: "agresión física» (categoría 13), "agresión verbal y gestual» (categoría 11), ser "tonto, niñato o inmaduro» (categoría 9), "caer mal» (categoría 3) y ser "pesado o molesto» (categoría 10). Existe otro bloque de motivos formado por categorías que agrupan un $5 \%$ de las respuestas de los niños, incluye: falta de «amistad »(categoría 1), «aburrido o retraído» (categoría 8) y "falta de relación» (categoría 15). Con pocas respuestas, aparecen las categorías: "comportamiento antisocial o vandalismo" (categoría 14), «antipatía» (categoría 5), «mal compañero» (categoría 2), «mala competencia académica» (categoría 7) y «mal carácter» (categoría 6). Finalmente, destacar que las "características físicas» (categoría 4) no son un motivo importante de rechazo en estas edades.

Recientemente se han estudiado los motivos de rechazo emitidos por niños de 6-8 años (Sanahuja, 2013). A partir de los 2.965 motivos emitidos se ha constituido una taxonomía que podemos ver en la Tabla 1. La cual nos explica y ordena la gran multiplicidad de motivos que emiten los niños.

Tabla 1. Categorías y subcategorías de rechazo (García Bacete y Sanahuja, 2013).

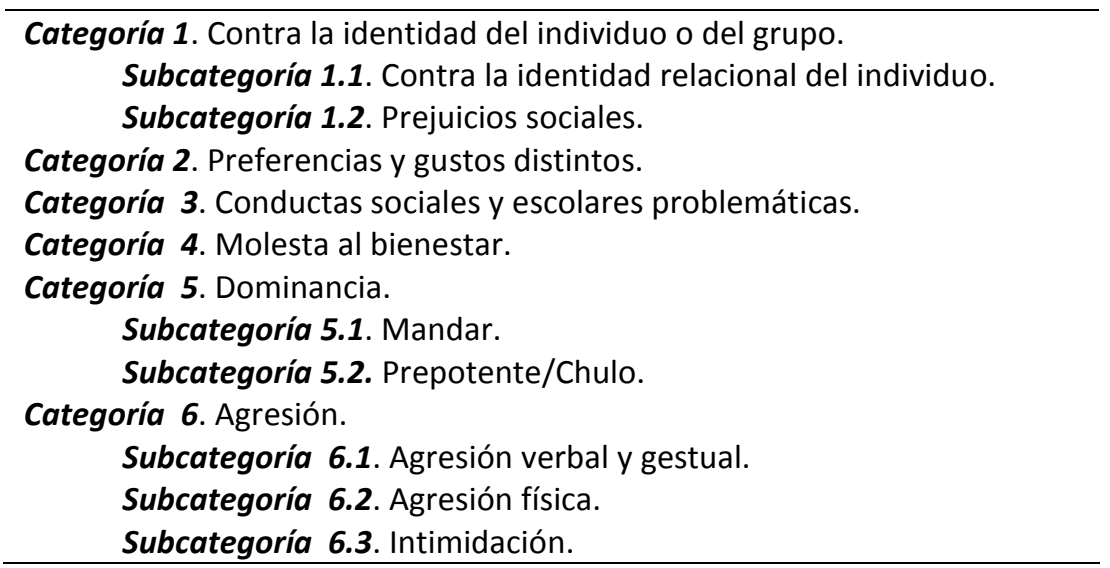

En la literatura consultada no se ha encontrado ningún estudio que haga referencia a la comparación de los motivos de rechazo de alumnos pertenecientes a diferentes zonas geográficas. Es por ello que este 
estudio se centra en las razones que emiten los alumnos del primer ciclo de educación primaria de Castellón, Palma de Mallorca, Sevilla y Valladolid, para mostrar su rechazo hacia sus compañeros.

\section{Objetivos}

El principal objetivo de este estudio es analizar la tasa de los motivos de rechazo y su distribución en función de la zona geográfica del emisor. De esta manera podremos conocer donde radican las principales diferencias en la emisión de motivo de rechazo por parte de los alumnos de Castellón, de Palma de Mallorca, de Sevilla y de Valladolid. Así mismo también sabremos qué motivos son más importantes para unos u otros alumnos.

\section{Material y método}

\section{Participantes}

En este estudio han participado un total de 939 alumnos del primer ciclo de educación primaria, escolarizados en 40 aulas de centros públicos de España en las ciudades de Castellón de la Plana, Palma de Mallorca, Sevilla y Valladolid. Más concretamente podemos decir que de todos los alumnos participantes 264 fueron de Castellón de la Plana (28,1\%), 296 de Palma de Mallorca (31,5 \%), 272 de Sevilla (29 \%) y 107 de Valladolid $(11,4 \%)$.

Por lo que respecta al género de los participantes decir que: 472 fueron niños (50,3\%) y 467 niñas (47,8\%). De las 40 aulas que han participado, 27 aulas pertenecían a 1 o de primaria $(\mathrm{N}=642 ; 68,4 \%)$ y 13 aulas a 2 o de primaria $(\mathrm{N}=297 ; 31,6 \%)$.

\section{Instrumentos}

El instrumento utilizado para la recogida de datos fue un cuestionario sociométrico de nominación entre iguales (GREI, 2009; publicado en García Bacete y González, 2010). Se trata de un sistema de nominaciones entre iguales que pone al niño en situación de elegir a compañeros de clase en función de un criterio positivo o negativo previamente establecido. Al alumnado se le preguntó: "De todos los niños y niñas de tu clase con quién te gusta estar más, ¿por qué?» y "De todos los niños y niñas de tu clase con quién te gusta estar menos, ¿por qué?», para establecer las nominaciones positivas y negativas. El número de nominaciones máximo era de 10 por cada opción. Es necesario matizar que en el presente trabajo solo se han analizado los motivos negativos, es decir, los motivos que los niños esgrimen para rechazar a sus compañeros. 


\section{Procedimiento}

Este estudio forma pare de una investigación más amplia que está Ilevando a cabo actualmente el equipo de investigación del GREI (Grupo interuniversitario de investigación del Rechazo entre Iguales en el contexto escolar). Es por ello que previamente al inicio de este estudio, ya se contaba con todas las autorizaciones necesarias de las instituciones educativas pertinentes, de los centros escolares y de las familias de los alumnos participantes.

Con el fin de alterar el mínimo posible la dinámica de la clase se acordó con los tutores de cada aula las fechas para realizar los pases. El "Cuestionario sociométrico de nominación entre iguale»s fue aplicado en cada aula por miembros del equipo de investigación del GREI (Grupo interuniversitario de investigación del Rechazo Entre Iguales en el contexto escolar) en sus respectivas sedes (Castellón de la Plana, Palma de Mallorca, Sevilla y Valladolid). Para este cuestionario, los niños disponían de una orla con las fotos de sus compañeros. Cuando se les formulaban las preguntas, los niños elegían a sus compañeros señalando con el dedo las fotos de los niños que nominaban. Además, se pidió que justificaran porque razón o motivo preferían o no estar con los niños que habían nominado positivamente o negativamente (García Bacete, Sánchiz y Marande, 2011).

Una vez se dispuso de todos los datos en formato papel se pasó al correspondiente vaciado y se inició el laborioso proceso de categorización siguiendo la propuesta de la teoría fundamentada - grounded theory (Carrero, 1999). Este proceso finalizó con el acuerdo interjueces, el cual oscilaba entre el "perfecto" y el "casi perfecto» según la interpretación de la estadística kappa (к) de Cohen. Así pues llegamos a la categorización que hemos presentado en la introducción. Después dado el acuerdo al que se llegó y viendo que las categorías estaban bien constituidas se pasó al vaciado de la codificación de cada motivo al SPSS para poder proceder con los análisis estadísticos.

Por lo que respecta a las estrategias de análisis, destacar que primeramente, se efectuó un "análisis de contenido" para llegar de los 2.965 motivos de rechazo a las 6 categorías y 7 subcategorías que explican el por qué del rechazo entre niños de 6-8 años. Para el análisis de datos se ha utilizado el programa SPSS Statistics 19. Los análisis estadísticos que se han realizado han sido: tablas de contingencias, en estadística se emplean para registrar y analizar la relación entre dos o más variables, las hemos utilizado para ver el acuerdo interjueces y los porcentajes de motivos distribuidos; el estadístico de contraste que hemos utilizado ha sido el Chi-Cuadrado (?2), el cual nos idnica el nivel de significación, es decir, nos informa si se superan los límites fijados en ciencia (en psicología $\mathrm{p}<.05$ ); y tamibén se han realizado pruebas $T$ para muestras relacionadas, para poder comprobar si los porcentajes obtenidos en una tabla de contingencia son significativos o no, es decir, para ver si existen diferencias estadísticas entre los porcentajes. Por 
ejemplo: vemos como la categoría 1, contra la identidad del individuo o del grupo obtiene un porcentaje de $13,5 \%$ y la categoría 3, conductas sociales y escolares problemáticas obtiene un porcentaje de $12,8 \%$. Vemos como a simple vista la categoría 1 recibe más porcentaje que la 2 pero la prueba $T$ para muestra relacionada nos indica que no existen diferencias estadísticas entre ambos valores, así pues ambas categorías conforman un mismo bloque de motivos.

\section{Resultados}

Los resultados obtenidos muestran diferencias significativas en función de la zona de procedencia, por tanto podemos afirmar que alumnos de Castellón, de Palma de Mallorca, de Sevilla y de Valladolid presentan un perfil diferente en la emisión de las razones de rechazo, ?] ?] ?] ${ }^{2}{ }_{(18)}=105.173 p \leq .000$. En la tabla 2, podemos ver la distribución de los motivos según la zona de procedencia.

Los alumnos de Castellón emiten un total de 1024 (34,5\%) motivos de rechazo, los de Palma de Mallorca emiten 816 (27,5\%) motivos de rechazo, los de Sevilla emiten $801(27,0 \%)$ motivos de rechazo y los de Valladolid emiten $324(10,9 \%)$ motivos de rechazo.

Tabla 2. Frecuencias y porcentajes de los motivos de rechazo por zona.

\begin{tabular}{|c|c|c|c|c|c|c|c|c|}
\hline \multirow[t]{2}{*}{ Categorías } & \multicolumn{2}{|c|}{ Castellón } & \multicolumn{2}{|c|}{ Palma de Mallorca } & \multicolumn{2}{|c|}{ Sevilla } & \multicolumn{2}{|c|}{ Valladolid } \\
\hline & $\%$ & $\begin{array}{c}\text { Residuos } \\
\text { tipificados } \\
\text { corregidos }\end{array}$ & $\%$ & $\begin{array}{c}\text { Residuos } \\
\text { tipificados } \\
\text { corregidos }\end{array}$ & $\%$ & $\begin{array}{c}\text { Residuos } \\
\text { tipificados } \\
\text { corregidos }\end{array}$ & $\%$ & $\begin{array}{c}\text { Residuos } \\
\text { tipificados } \\
\text { corregidos }\end{array}$ \\
\hline 0. No emiten ninguna razón de rechazo. & ,3 & $-2,6$ & 1,0 & 2 & 1,0 & ,3 & 2,5 & 3,1 \\
\hline 1. Contra la identidad del individuo o del grupo. & 13,5 & 1,7 & 14,3 & 2,4 & 7,9 & $-4,3$ & 12,0 & 0 \\
\hline $\begin{array}{l}\text { 1.1. Contra la identidad relacional del } \\
\text { individuo. }\end{array}$ & 6,9 & $-1,1$ & 9,6 & 2,4 & 5,6 & $-2,6$ & 10,5 & 2,0 \\
\hline 1.2. Prejuicios sociales. & 6,5 & 4,3 & 4,8 & ,7 & 2,2 & $-3,4$ & 1,5 & $-2,6$ \\
\hline 2. Preferencias y gustos distintos. & 18,2 & 5,0 & 9,2 & $-4,5$ & 12,6 & $-1,2$ & 14,8 & ,5 \\
\hline 3. Conductas sociales y escolares problemáticas. & 12,8 & $-3,2$ & 15,0 &,- 7 & 21,3 & 5,1 & 13,3 & $-1,3$ \\
\hline 4. Molesta al bienestar. & 23,0 & 2,9 & 20,5 & 3 & 16,6 & $-2,9$ & 18,2 & -9 \\
\hline 5. Dominancia. & 4,8 & 4 & 4,4 &,- 2 & 4,6 & 1 & 4,0 &,- 5 \\
\hline 5.1. Mandar. & 4,4 & 6 & 3,8 &,- 5 & 4,1 & 1 & 3,7 &,- 4 \\
\hline 5.2. Prepotente/Chulo. & 4 &,- 5 & 6 & ,7 & 5 & 1 & ,3 &,- 5 \\
\hline 6. Agresión. & 27,4 & $-4,6$ & 35,7 & 2,0 & 36,0 & 2,2 & 35,2 & 9 \\
\hline 6.1. Agresión verbal y gestual. & 4,1 & 9 & 3,4 &,- 4 & 3,4 &,- 5 & 3,7 & 0 \\
\hline 6.2. Agresión física. & 22,2 & $-2,3$ & 26,5 & 1,4 & 23,7 &,- 7 & 29,9 & 2,4 \\
\hline 6.3. Intimidación. & 1,2 & $-6,4$ & 5,8 & 1,9 & 8,9 & 6,9 & 1,5 & $-2,8$ \\
\hline TOTAL & 100 & & 100 & & 100 & & 100 & \\
\hline
\end{tabular}

Vemos como los motivos más frecuentes que dan los alumnos de Castellón para manifestar su rechazo son: en primer lugar por la agresión $(27,4 \%)$ y más concretamente la agresión física. En segundo lugar por molestar al bienestar (23,0\%). En tercer lugar por preferencias y gustos distintos (18,2\%). Cuarto lugar por presentar motivos que atentan contra la identidad del individuo o del grupo $(13,5 \%)$ y conductas sociales y 
escolares problemáticas (12,8\%). En quinto lugar por la de dominancia $(4,8 \%)$.

Por otra parte, los motivos más frecuentes que dan alumnos de Palma de Mallorca para manifestar su rechazo son: primero por la agresión (35,7\%) y más concretamente la física. Segundo por molestar al bienestar (20,5\%). Tercero por conductas sociales y escolares problemáticas $(15,0 \%)$ y por motivos que van contra la identidad del individuo o del grupo (14,3\%). Cuarto por preferencias y gustos distintos $(9,2 \%)$. Quinto por la dominancia $(4,4 \%)$.

Los motivos más frecuentes que dan los alumnos de Sevilla para manifestar su rechazo son: en primer lugar la agresión $(36,0 \%)$ y más concretamente la física. En segundo lugar conductas sociales y escolares problemáticas (21,3\%). En tercer lugar molestar al bienestar (16,6\%). En cuarto lugar por preferencias y gustos distintos (12,6\%). En quinto lugar contra la identidad del individuo o del grupo (7,9\%). Finalmente vemos como la categoría menos nombrada es la de dominancia $(4,6 \%)$.

Por último, los motivos más frecuentes que dan los alumnos de Valladolid para manifestar su rechazo son: en primer lugar por la agresión $(35,2 \%)$ y más concretamente la física. En segundo lugar por molestar al bienestar (18,2\%). En tercer lugar por preferencias y gustos distintos $(14,8 \%)$, conductas sociales y escolares problemáticas $(13,3 \%)$ y contra la identidad del individuo o del grupo (12,0\%). Finalmente por la dominancia (4,0\%).

Fijándonos en los residuos tipificados corregidos, vemos como los alumnos de Palma de Mallorca son los que más motivos referentes a contra la identidad del individuo o del grupo emiten, mientras que los alumnos de Sevilla son los que menos motivos emiten de dicha categoría. Centrándonos ahora en las subcategorías de la categoría 1, vemos como los alumnos de Palma de Mallorca y Valladolid son los que más motivos emiten de la subcategoría 1.1 (contra la identidad relacional del individuo), y los alumnos de Sevilla son los que menos motivos emiten. Los alumnos de Castellón comparado con los alumnos de todas las demás zonas emiten más motivos de rechazo referentes a prejuicios sociales, y los que menos Sevilla y Valladolid. En Castellón también se emiten más motivos referentes a preferencias y gustos distintos y los que menos motivos emiten de esta categoría son los alumnos de Palma de Mallorca. Los alumnos que más motivos emiten relacionados con conductas sociales y escolares problemáticas son los de Sevilla y los que menos los de Castellón. Los alumnos que más motivos emiten por molestar al bienestar son los de Castellón y los que menos Sevilla. Por último remarcar que los alumnos de Castellón son los que menos motivos emiten referentes a la agresión y los que más son los alumnos de Sevilla y Palma de Mallorca. Por lo que respecta a la agresión física los alumnos de Valladolid son los que más motivos emiten de dicha subcategoría y los que menos son los de Castellón. En cuanto a la intimidación los alumnos de Sevilla son los que más motivos emiten de dicha subcategoría y los que menos son los de Castellón y los de Valladolid. 


\section{Discusión y conclusiones}

Los resultados obtenidos muestran diferencias significativas en función de la zona de procedencia, podemos afirmar que alumnos de Castellón, de Palma de Mallorca, de Sevilla y de Valladolid presentan un perfil diferente en la emisión de razones de rechazo, esto puede darse porque existen diferencias culturales entre las diferentes zonas. En la tabla 3, pasamos a comparar el orden más frecuente de motivos emitidos por alumnos de cada zona.

Tabla 3. Orden de motivos emitidos por los alumnos de cada zona.

\begin{tabular}{|c|c|c|c|c|}
\hline $\begin{array}{l}\text { Orden de motivos más } \\
\text { frecuentes. }\end{array}$ & Castellón & Palma de Mallorca & Sevilla & Valladolid \\
\hline 10 & 〜Agresión. & 〜Agresión. & 〜Agresión. & 〜Agresión. \\
\hline 20 & $\sim$ Molestar al bienestar. & $\sim$ Molestar al bienestar. & $\begin{array}{l}\sim \text { Conductas sociales } \quad \text { y } \\
\text { escolares problemáticas. }\end{array}$ & $\sim$ Molestar al bienestar. \\
\hline 30 & $\begin{array}{l}\sim \text { Preferencias y gustos } \\
\text { distintos. }\end{array}$ & $\begin{array}{l}\text { 〜Conductas sociales y } \\
\text { escolares problemáticas. } \\
\sim \text { Contra la identidad del } \\
\text { individuo o del grupo. }\end{array}$ & $\sim$ Molestar al bienestar. & $\begin{array}{l}\sim \text { Preferencias y gustos } \\
\text { distintos. } \\
\sim \text { Conductas sociales y } \\
\text { escolares problemáticas. } \\
\sim \text { Contra la identidad del } \\
\text { individuo o del grupo. }\end{array}$ \\
\hline 40 & $\begin{array}{l}\sim \text { Contra la identidad del } \\
\text { individuo o del grupo. } \\
\sim \text { Conductas sociales y } \\
\text { escolares problemáticas. }\end{array}$ & $\begin{array}{l}\sim \text { Preferencias y } \text { gustos } \\
\text { distintos. }\end{array}$ & $\begin{array}{lll}\sim \text { Preferencias } & \text { y } & \text { gustos } \\
\text { distintos. } & & \\
\end{array}$ & 〜Dominancia. \\
\hline 50 & $\sim$ Dominancia. & $\sim$ Dominancia. & $\begin{array}{l}\text { Contra la identidad del } \\
\text { individuo o del grupo. }\end{array}$ & \\
\hline 60 & & & $\sim$ Dominancia. & \\
\hline
\end{tabular}

Vemos como para todos los niños, de las diferentes zonas geográficas, el principal motivo de rechazo es la agresión. No obstante cabe destacar que los alumnos de Castellón son los que menos alusión hacen a la agresión y los que más son los alumnos de Sevilla y Palma de Mallorca.

En segundo lugar vemos como, para los alumnos de Castellón, Palma de Mallorca y Valladolid, el segundo bloque de motivos más importante por el que rechazan a sus iguales hacen referentes a molestar al bienestar. Remarcar que los alumnos de Castellón son los que más motivos de dicha categoría emiten. Destacar que para los alumnos de Sevilla el segundo bloque de motivos más importante son los referentes a conductas sociales y escolares problemáticas.

En tercer lugar, remarcar que para los alumnos de Castellón el tercer bloque de motivos en relaciona a su importancia son los referentes a preferencias y gustos distintos. Por lo que respecta a los alumnos de Palma de Mallorca notemos como en tercer lugar los motivos por los que estos alumnos rechazan hacen referencia a conductas sociales y escolares problemáticas y por motivos que van contra la identidad del individuo o del grupo. Los alumnos de Sevilla, en tercer lugar, rechazan por motivos referentes a molestar al bienestar. Y los alumnos de Valladolid, en tercer lugar, rechazan por: preferencias y gustos distintos, conductas sociales y escolares problemáticas y por motivos que van contra la identidad del 
individuo y del grupo.

En cuarto lugar, vemos como los alumnos de Castellón rechazan por motivos que van contra la identidad del individuo o del grupo, y por conductas sociales y escolares problemáticas. Los alumnos de Palma de Mallorca y los alumnos de Sevilla rechazan, en cuarto lugar, por motivos referentes a preferencias y gustos distintos. En cuarto y último lugar, los alumnos de Valladolid rechazan por motivos referentes a la dominancia.

En quinto lugar, notemos como los alumnos de Castellón y de Palma de Mallorca rechazan por motivos referentes a la dominancia. Y los alumnos de Sevilla, en quinto lugar, rechazan con motivos que van contra la identidad del individuo o del grupo.

En sexto lugar los alumnos de Sevilla rechazan con motivos que aluden a la dominancia. Así como primeramente hemos destacado que todos los alumnos de las diferentes zonas rechazan principalmente por motivos referentes a la agresión, podemos finalizar diciendo que el bloque de motivos menos utilizado por los niños de las diferentes zonas son aquellos que hacen referencia la dominancia.

Así pues podemos concluir con este estudio que los alumnos de diferentes zonas emiten diferentes motivos de rechazo para rechazar a sus iguales, esto se puede deber a las diferencias culturales y sociales que existen entre las diferentes zonas geográficas.

\section{Bibliografía}

BukoWski, W. M., NeWcomB, A. F. y HaRTUP, W. W. (1996): The company they keep: Friendship in childhood and adolescence. New York, EE.UU.: Cambridge University Press.

CARrero, V. (1999): Análisis cualitativo de datos: aplicación de la teoría fundamentada ("Grounded Theory") en el ámbito de la innovación organizacional. Universitat Jaume I de Castelló. Publicacions de la UJI, ed. II. Titol. III. Sèrie. (043.2)(460.311).

GARCíA BACETE, F. J y GonzÁlez, J. (2010): Evaluación de la competencia social entre iguales. La sociometría y otras medidas. Madrid: TEA Ediciones.

García BACETE, F. J., SUREDA, I., y Monjas I. (2010): «El rechazo entre iguales en la educación primaria: Una panorámica general». Anales de Psicología, $26,1,123-136$.

García BACETE, F.J., SÁNChiz, M. L., y MARANDE, G. (2011): «Sociomet: Una herramienta para el estudio sociométrico».

SÁNCHIZ, M. L., M. Martí, I. Cremades (Eds.): «Orientación e intervención educativa. Retos para los orientadores del siglo XXI» (pp 707-716).Tirant lo Blanc. Valencia.

GRUPO INTERUNIVERSITARIO DE INVESTIGACIÓN DEL RECHAZO ENTRE IGUALES EN EL CONTEXTO ESCOLAR (GREI) (2009): Cuestionario Sociométrico de 
nominaciones entre iguales. Memória de investigación 2009. Universitat Jaume I.

HartuP, W.W. (1983): Peer relations. En P.H. Mussen (Series Ed.) y Hetherington (Vol. Ed.), «Handbook of child psychology: Vol.4 Socializations, personality, and social development»(4a ed., pp. 102-196). Nueva York: Wiley.

Monjas, I. (2007) (Dir): Cómo promover la convivencia: Programa de asertividad y habilidades sociales (PHAS). Madrid: CEPE.

Monjas, I., Sureda, I., y García BACETE, F. J., (2008): "¿Por qué los niños y niñas se aceptan y se rechazan?»Cultura y Educación,20, 4, 479-492.

RogosCH, F.A. Y Newcombs, A.F. (1989): «Children's Perceptions of Peer Reputations and Their Social Reputations among Peers». Child Development, 60, 597-610.

SANAHUja, A. (2013): Necesidades básicas y motivos de rechazo de los niños y niñas del primer ciclo de educación primaria. (Tesina final de máster inédita). Universitat Jaume I. Castellón.

Sureda, I., García Bacete, F. J. y Monjas I., (2009): "Razones de niños y niñas de diez y once años para preferir o rechazar a sus iguales». Revista latinoamericana de psicología, 41, 2, 305-321.

TODD, C. M (Ed.): Day care center connec-tions, 3, 1, (pp. 3-5). UrbanaChampaign, IL: University of Illinois Cooperative Extension Service. National Network for Child Care.

Williams, G. y Asher, S. R. (1993): Children without friends, Part 2: The reasons for peer rejection. 